\title{
Berlin erwartet Sie!
}

A us gutem Grund hatten sich vor einigen Jahren die drei großen Fachgesellschaften, die Deutsche Gesellschaft für Allergologie und klinische Immunologie (DGAKI), die Gesellschaft für Pädiatrische Allergologie und Umweltmedizin (GPA) sowie der Ärzteverband Deutscher Allergologen (ÄDA) entschlossen, gemeinsam einen großen deutschen Allergiekongress auszurichten. Die letzten drei Jahre haben gezeigt, dass dieses Konzept auf große Sympathie bei Wissenschaftlern in der allergologischen Forschung sowie bei allergologisch tätigen Ärzten aus Klinik und Praxis stößt.

Der 4. Gemeinsame Deutsche Allergiekongress wird vom 3. bis zum 6 . September 2009 in der Bundeshauptstadt Berlin stattfinden. Als Kongressort haben die Veranstalter das MaritimHotel Berlin ausgewählt, welches geradezu ideale Voraussetzungen für die wichtigste allergologische Veranstaltung im deutschsprachigem Raum bietet: Es ist leicht erreichbar vom Hauptbahnhof und Flughafen und liegt zentral am Kulturforum in unmittelbarer Nähe des Potsdamer Platzes und des Tiergartens. Die Möglichkeit, im Kongresshotel zu

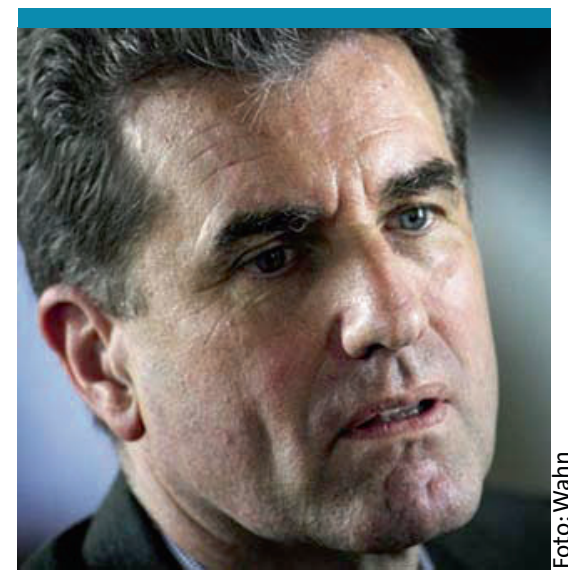

Prof. Dr. Ulrich Wahn, Klinik für Pädiatrie mit Schwerpunkt Pneumologie und Immunologie, Charité, Berlin

tagen und zu wohnen, eröffnet ausgezeichnete Möglichkeiten für Treffen unter Kollegen am Rande der Veranstaltung sowie für unkomplizierte Kontakte mit Experten und Repräsentanten der Industrie.

Das wissenschaftliche Programmkomitee hat in den letzten Monaten intensiv gearbeitet, um Plenarsitzungen, Symposien und Meet-the-Profes-
sor-Seminare auf höchstem Niveau zusammenzustellen und erstklassige Wissenschaftler und Kliniker aus dem deutschsprachigen Raum sowie aus anderen europäischen Ländern als Referenten nach Berlin zu holen. Gesonderte Veranstaltungen für medizinisches Assistenzpersonal werden ganz sicher auf großes Interesse stoßen. Darüber hinaus dürfen Sie ein abwechslungsreiches und unterhaltsames Gesellschaftsprogramm erwarten. Melden Sie sich rasch zum 4. Deutschen Allergiekongress an und denken Sie daran, frühzeitig auch Ihr Hotel in Berlin zu buchen. Wir erwarten eine Rekordteilnehmerzahl und hoffen sehr, dass die Kongresstage für Sie zu einem anregenden und für Ihre Arbeit bedeutsamen Kongressereignis werden.

Ich freue mich, Sie beim 4. Deutschen Allergiekongress zu sehen. Berlin erwartet Sie!

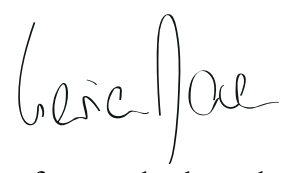

Prof. Dr. Ulrich Wahn

Kongresspräsident

\section{Gemeinsamer Deutscher Allergiekongress auf einen Blick \\ Veranstalter \\ AKM Allergiekongress und Marketing GmbH, Röntgenstraße 6a, 53177 Bonn-Bad Godesberg für \\ Ärzteverband Deutscher \\ Allergologen (ÄDA) \\ Deutsche Gesellschaft für Allergologie und klinische Immunologie (DGAKI) \\ Gesellschaft für Pädiatrische Allergologie und Umwelt- medizin (GPA)

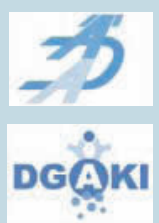 \\ Kongresstermin \\ 3.-6. September 2009 \\ Kongressort \\ Maritim Hotel, Stauffenbergstraße 26, 10785 Berlin \\ Kongresspräsidentschaft \\ Prof. Dr. Ulrich Wahn \\ Kongresssekretariat \\ Dr. Kirsten Beyer, Klinik für Pädiatrie m.S. Pneumologie und Immunologie, Charité, Augustenburger Platz 1, 13353 Berlin Tel.: (о 30 ) 4505 66-1 31 \\ Fax: (0 30) 4505 66-9 31 \\ E-Mail: kirsten.beyer@charite.de \\ Kongressorganisation und Auskunft \\ Ivo van der Ree, Flaskamp AG, Klosterstraße 64,10179 Berlin \\ Tel.: (० зо) 4 60 06-7 02 \\ Fax: (0 30) 460 06-7 70 \\ E-Mail: allergiekongress@flaskamp.de \\ Abstracteinreichung \\ Wissenschaftliche Beiträge können bis zum 15. Juni 2009 unter http://abstracts.urban- vogel.de online eingereicht werden.

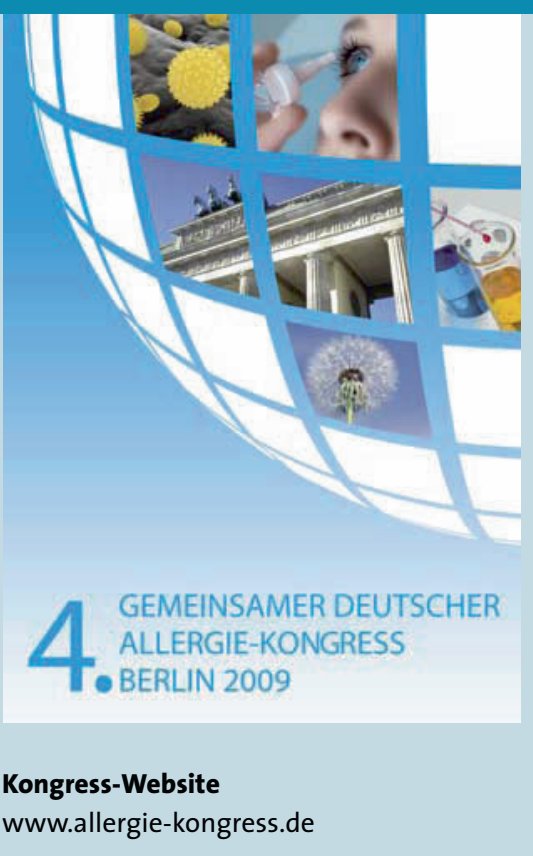

